\title{
Temporal trends in the biomass of three epibenthic invertebrates from the deep-sea observatory HAUSGARTEN (Fram Strait, Arctic Ocean)
}

\author{
J. Taylor, B. Staufenbiel, T. Soltwedel, M. Bergmann* \\ Alfred-Wegener-Institut, Helmholtz-Zentrum für Polar- und Meeresforschung, Am Handelshafen 12, 27570 Bremerhaven, \\ Germany
}

\begin{abstract}
In a bid to further understand processes that influence deep-sea epibenthic megafauna, which fulfil critical roles in the global carbon cycle, we present data from the Arctic Long-Term Ecological Research observatory HAUSGARTEN, in the Fram Strait, showing significant temporal changes in total biomass of 3 key organisms (Kolga hyalina, Elpidia heckeri and Mohnia spp.) at stations N3, HG-IV and S3 during repeated deployments over a time series spanning 2004-2015. Overall, all species investigated displayed a similar reproduction/recruitment cycle, with increasing mean mass per individual leading to decreases in abundance, and vice versa. However, there were 3 'events' that deviated from this pattern. The first was a mass reproduction event of E. heckeri at HG-IV from 2012 onwards, likely due to an increased carrying capacity. The second event involved migration of $K$. hyalina from HG-IV between 2004-2007, with a return in 2011. This coincided with a shift in the composition of the particle flux at the station. The final event was a mass migration of $K$. hyalina to N3 between $2004\left(0\right.$ ind. $\left.\mathrm{m}^{-2}\right)$ and 2007 $\left(4.765 \pm 0.084\right.$ ind. $\left.\mathrm{m}^{-2}\right)$. This event coincided with a 4 -fold increase in phytodetrital food availability at the seafloor at N3. Our results highlight the importance of time-series studies to ascertain the key factors that influence epibenthic megafaunal communities. They also highlight the fact that more needs to be done in understanding the life history of these organisms, as this understanding is, so far, widely lacking.
\end{abstract}

KEY WORDS: Arctic · Biomass - Deep-sea · Megafauna $\cdot$ HAUSGARTEN · Image analysis · Time-series

\section{INTRODUCTION}

Epibenthic megafauna, those individuals $\geq 1.5 \mathrm{~cm}$ (Grassle et al. 1975), inhabit the sediment-water interface almost exclusively. With the world's oceans acting as the largest carbon sink it is critical to understand these deep-sea organisms, their interactions with the environment and the drivers of change in their populations, as through their role as ecosystem engineers they are involved with the redistribution of organic matter from the seafloor via bioturbation, oxygenation and remineralisation (Ruhl, 2007, FitzGeorge-Balfour et al. 2010, Buhl-Mortensen et al.

${ }^{*}$ Corresponding author: Melanie.Bergmann@awi.de
2016). Determining standing stock biomass of a species can provide important information on the carbon budgets of a system. Traditionally, the assessment of deep-sea epibenthic megafaunal biomass or body length, which can be considered a proxy for biomass, has been defined through the deployment of trawling and sledge gears. Such methods have been used to observe spatio-temporal variations in non-crustacean bathyal invertebrates in the Catalan Sea (RamírezLlodra et al. 2007), to gauge temporal variations in body size of the sea cucumber Amperima rosea at the Porcupine Abyssal Plain (PAP) (Billett et al. 2001), to assess carbon flow in the benthic food web at PAP

() The authors 2018. Open Access under Creative Commons by Attribution Licence. Use, distribution and reproduction are unrestricted. Authors and original publication must be credited. 
(van Oevelen et al. 2012) and in the Gulf of Mexico (Rowe et al. 2008), to determine the effects of surface primary production on the biomass of invertebrate megabenthos on the Mid-Atlantic Ridge (Alt et al. 2013), and to compare deep-sea respiration with community dynamics at Station M, west of California, and PAP (Ruhl et al. 2014). The use of trawling and sledge equipment is destructive by nature and only semi-quantitative, potentially leading to order of magnitude underestimations of epibenthic megafaunal standing stock biomass in a given area (Durden et al. 2016). Therefore, whilst it is an important initial step to obtain specimens for the taxonomic identification of species and to allow for the determination of the presently lacking conversion factors for specific species, the use of camera-based surveys are key to understanding spatial and temporal trends in biomass. Previous studies that used camera-based assessments for megafaunal biomass estimates have typically looked at whole communities with an emphasis on spatial rather than temporal variability (Piepenburg \& Schmid 1996, Hargrave et al. 2004, Rowe et al. 2008, Durden et al. 2015, 2016).

Such studies on Arctic benthic communities are few and far between, because of the technological and logistical constraints imposed by ice coverage. Previous temporal megafaunal community studies at the Arctic Long-Term Ecological Research (LTER) observatory HAUSGARTEN have taken place at stations HG-IV (Bergmann et al. 2011), HG-I (Meyer et al. 2013), N3 and S3 (Taylor et al. 2017), over varying time-spans, using abundance data obtained via the analysis of photographs from a towed camera system. Large temporal variations in the abundances of the deepsea holothurians Kolga hyalina and Elpidia heckeri and the gastropods Mohnia danielsseni and Mohnia mohni (hereafter referred to as Mohnia spp.) have previously been reported (Bergmann et al. 2011, Taylor et al. 2017). Billett \& Hansen (1982) and Billett (1991) suggest that deep-sea holothurians display a propensity to be opportunistic in nature, capable of utilising increased food input through rapid reproduction, often leading to patchy distributions. Little is known about the life history and reproductive strategies of Mohnia spp. Being mobile organisms, all 3 play a role as ecosystem engineers. As deposit feeders, which ingest sediment particles, this role is likely greater in the 2 sea cucumber species. However, Mohnia spp. also contribute to bioturbation as they create traces or 'Lebensspuren' when crawling over the soft sediments or burrowing for shelter.

Here, we produce the first assessments of biomass for these 3 numerically important species at the
HAUSGARTEN observatory as well as establishing conversion factors using the relationship between measured body length and preserved wet-weight relationships. We also address specific questions: (1) Can we derive sufficient conversion factors to estimate temporal variations in biomass of the 3 species through the use of sampled specimens? (2) Are there temporal variations in the overall biomass for each species at each station and how do they relate to variations in abundances? (3) Is there a relationship between abundance and mean mass per individual for the 3 species? Ultimately, we address what these questions imply for the life strategies of these species, with the relationships between biomass and abundances providing insight into behaviour with changing ecosystem dynamics.

\section{MATERIALS AND METHODS}

\section{Study location}

Established in 1999, the Arctic LTER observatory HAUSGARTEN currently comprises 21 permanent sampling stations along a latitudinal and a bathymetric gradient in the Fram Strait (Fig. 1; Soltwedel et al. 2016), representing the only connection for the exchange of deep and intermediate water masses between the north Atlantic and central Arctic Ocean (von Appen et al. 2015). The hydrography at the studied sites in the eastern part of the Fram Strait are characterised by the inflow of relatively warm, nutrient-rich water into the central Arctic Ocean (BeszczynskaMöller et al. 2012). Our study focuses on the northern (N3), central (HG-IV) and the southernmost (S3) stations, which form the latitudinal transect and are situated between 2351 and $2788 \mathrm{~m}$ water depth. The bottom topography at HG-IV is different to the other 2 stations, with an incremental slope towards the latter 2 thirds of the transect (Taylor et al. 2016). To account for this, only images from the first third of each photographic transect have been used for this study.

The station S3 remains mostly ice-free year round. By contrast, HG-IV and especially N3 experience ice coverage to varying degrees (Taylor et al. 2016, 2017). Melting of sea-ice in spring and summer contributes to a stratified, nutrient rich, marginal ice zone, causing intense phytoplankton blooms and regionally enhanced fluxes of particulate organic matter (Bauerfeind et al. 2009, Lalande et al. 2013). Consistent sampling campaigns and long-term deployments of moorings and free-falling systems have yielded a comprehensive data set comprising bacterial, biogeo- 


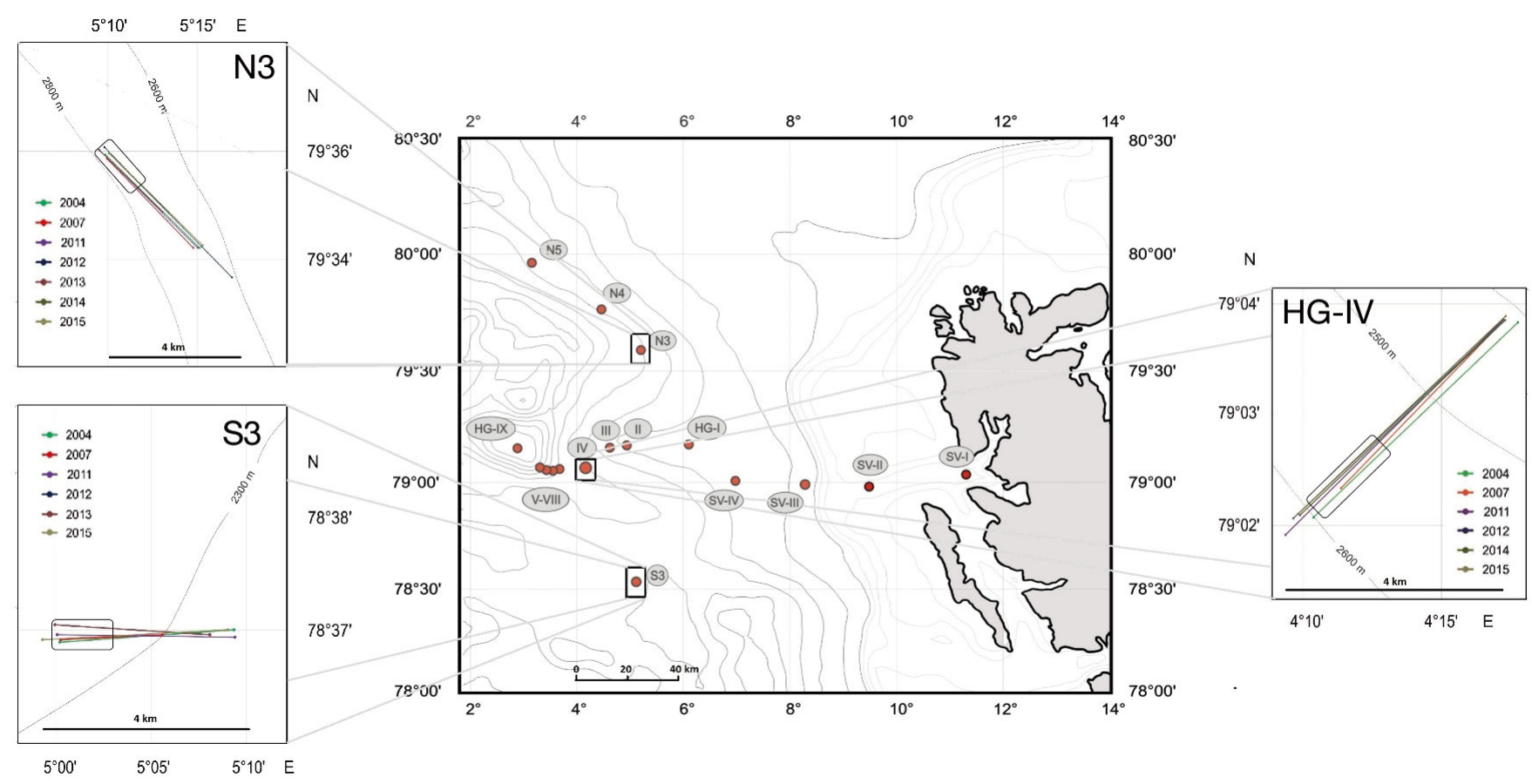

Fig. 1. LTER observatory HAUSGARTEN in Fram Strait, and the location of the camera transects conducted at stations N3, HG-IV and S3 from 2004 to 2015. Rectangle over transects depicts area section of the images used

chemical and faunal data, as well as hydrographical data, sedimentation patterns and geological properties. This allows for greater potential of understanding the wide variety of linked systems and factors contributing to their changes (e.g. Bauerfeind et al. 2009, Forest et al. 2010, Hasemann \& Soltwedel 2011, Jacob et al. 2013, Górska et al. 2014, Meyer et al. 2016, Soltwedel et al. 2016).

\section{Deployment of sampling gears}

Seafloor images of the 3 transect stations analysed during this study were obtained during the expeditions ARK-XX/1 (2004), ARK-XXII/2 (2007), ARKVI/2 (2011), ARK-XXVII/2 (2012), ARK-XXVIII/1 (2014) and ARK XXIX/2 (2015) aboard the German research icebreaker 'Polarstern' and MSM29 (2013) aboard RV 'Maria S. Merian'. During the 2004 and 2007 campaigns, an analogue Ocean Floor Observation System (OFOS) was chartered from Oktopus, Germany. From 2011 onwards, the Alfred Wegener Institute (AWI) has used its own digital OFOS (Taylor et al. 2017).

To obtain seafloor images of the 3 transects the OFOS was towed at each station for 4 hours at ca. 0.5 knots to cover a distance of $4 \mathrm{~km}$ at a target altitude of $1.5 \mathrm{~m}$. The altitude was controlled, under instruction, by a winch operator, to maintain target altitude, reacting to changes in seafloor topography and sea state. The still camera was triggered automatically at 30 -s intervals to avoid spatial overlap of images, as well as observer bias.

The biogeochemical sediment parameters were obtained as part of the continual LTER HAUSGARTEN programme conducted by the AWI. Virtually undisturbed sediment samples were taken in parallel at each station using a video-guided multiple corer (MUC). Cores were sub-sampled 3-4 times (only once for particulate organic carbon from 2004-2008) using plastic syringes ( 1 and $2 \mathrm{~cm}$ diameter) modified with the anterior ends cut off. Results from the uppermost $1 \mathrm{~cm}$ were used in this study. The majority of pigments (chloroplastic pigment equivalents, CPE) indicate food availability from photosynthetically derived material reaching the seafloor. They were extracted in $90 \%$ acetone and measured by a Turner fluorometer (Yentsch \& Menzel 1963). Organic carbon contents of the sediments were determined using a LECO CS125 carbon analyser, following a method by Bernard et al. (2004). Phospholipids, representative for the total microbial biomass, were analysed photometrically according to Findlay et al. (1989). Particulate proteins (readily soluble per sediment volume), indicative of living and dead biomass (organisms and detrital matter within the sediments) were determined following a method by Greiser \& Faubel (1988). Table 1 details all MUC deployments. 
Table 1. Summary of gear deployments done at HAUSGARTEN stations N3, HG-IV and S3. OFOS: Ocean Floor Observation System; MUC: multiple corer; AGT: Agassiz trawl

\begin{tabular}{|c|c|c|c|c|c|c|c|c|}
\hline Cruise & $\begin{array}{l}\text { Station } \\
\text { number }\end{array}$ & $\begin{array}{l}\text { Station } \\
\text { name }\end{array}$ & $\begin{array}{c}\text { Date } \\
(\mathrm{dd} / \mathrm{mm} / \mathrm{yr})\end{array}$ & $\begin{array}{l}\text { Latitude } \\
\text { (N) }\end{array}$ & $\begin{array}{l}\text { Longitude } \\
\text { (E) }\end{array}$ & $\begin{array}{l}\text { Depth } \\
\text { (m) }\end{array}$ & Gear & $\begin{array}{c}\text { No. of images } \\
\text { analysed }\end{array}$ \\
\hline ARK-XVIII/1 & PS62/161-2 & HG-IV & 02/08/2002 & $79^{\circ} 03.90^{\prime}$ & $4^{\circ} 10.93^{\prime}$ & 2469 & MUC & \\
\hline ARK-XVIII/1 & PS62/189-2 & S3 & 10/08/2002 & $78^{\circ} 34.97^{\prime}$ & $5^{\circ} 04.21^{\prime}$ & 2344 & MUC & \\
\hline ARK-XVIII/1 & PS62/192-2 & N3 & $11 / 08 / 2002$ & $79^{\circ} 35.02^{\prime}$ & $5^{\circ} 15.33^{\prime}$ & 2668 & MUC & \\
\hline ARK-XIX/3 & PS64/429-1 & HG-IV & $26 / 07 / 2003$ & $79^{\circ} 04.31^{\prime}$ & $4^{\circ} 07.57^{\prime}$ & 2501 & MUC & \\
\hline ARK-XIX/3 & PS64/453-1 & S3 & $30 / 07 / 2003$ & $78^{\circ} 36.50^{\prime}$ & $5^{\circ} 04.32^{\prime}$ & 2343 & MUC & \\
\hline ARK-XX/1 & PS66/106-1 & S3 & 08/07/2004 & $78^{\circ} 36.97^{\prime}$ & $5^{\circ} 00.33^{\prime}$ & 2363 & OFOS (start) & \\
\hline ARK-XX/1 & PS66/106-1 & S3 & 08/07/2004 & $78^{\circ} 37.02^{\prime}$ & $5^{\circ} 09.90^{\prime}$ & 2350 & OFOS (end) & 184 \\
\hline ARK-XX/1 & PS66/108-1 & S3 & 08/07/2004 & $78^{\circ} 37.50^{\prime}$ & $5^{\circ} 03.16^{\prime}$ & 2349 & MUC & \\
\hline ARK-XX/1 & PS66/117-1 & HG-IV & 09/07/2004 & $79^{\circ} 05.00^{\prime}$ & $4^{\circ} 04.98^{\prime}$ & 2508 & MUC & \\
\hline ARK-XX/1 & PS66/118-1 & HG-IV & 09/07/2004 & $79^{\circ} 09.75^{\prime}$ & $3^{\circ} 52.21^{\prime}$ & 2377 & AGT (start) & \\
\hline ARK-XX/1 & PS66/117-1 & HG-IV & 09/07/2004 & $79^{\circ} 09.50^{\prime}$ & $3^{\circ} 54.53^{\prime}$ & 2425 & AGT (end) & \\
\hline ARK-XX/1 & PS66/120-1 & HG-IV & 09/07/2004 & $79^{\circ} 01.99^{\prime}$ & $4^{\circ} 10.27^{\prime}$ & 2626 & OFOS (start) & \\
\hline ARK-XX/1 & PS66/120-1 & HG-IV & $10 / 07 / 2004$ & $79^{\circ} 03.87^{\prime}$ & $4^{\circ} 17.71^{\prime}$ & 2406 & OFOS (end) & 174 \\
\hline ARK-XX/1 & PS66/127-2 & N3 & $11 / 07 / 2004$ & $79^{\circ} 35.93^{\prime}$ & $5^{\circ} 09.50^{\prime}$ & 2791 & MUC & \\
\hline ARK-XX/1 & PS66/127-3 & N3 & $11 / 07 / 2004$ & $79^{\circ} 35.99^{\prime}$ & $5^{\circ} 10.56^{\prime}$ & 2784 & MUC & \\
\hline ARK-XX/1 & PS66/127-4 & N3 & $11 / 07 / 2004$ & $79^{\circ} 35.90^{\prime}$ & $5^{\circ} 09.93^{\prime}$ & 2788 & OFOS (start) & \\
\hline ARK-XX/1 & PS66/127-4 & N3 & $12 / 07 / 2004$ & $79^{\circ} 34.10^{\prime}$ & $5^{\circ} 15.18^{\prime}$ & 2661 & OFOS (end) & 182 \\
\hline ARK-XXI/2 & PS68/238-3 & HG-IV & $19 / 08 / 2005$ & $79^{\circ} 03.91^{\prime}$ & $4^{\circ} 10.81^{\prime}$ & 2462 & MUC & \\
\hline ARK-XXI/2 & PS68/247-1 & HG-V & $20 / 08 / 2005$ & $79^{\circ} 05.67^{\prime}$ & $3^{\circ} 38.68^{\prime}$ & 3072 & AGT (start) & \\
\hline ARK-XXI/2 & PS68/247-1 & $\mathrm{HG}-\mathrm{V}$ & $20 / 08 / 2005$ & $79^{\circ} 06.32^{\prime}$ & $3^{\circ} 38.78^{\prime}$ & 3102 & AGT (end) & \\
\hline ARK-XXI/2 & PS68/250-2 & N3 & $21 / 08 / 2005$ & $79^{\circ} 36.23^{\prime}$ & $5^{\circ} 10,32^{\prime}$ & 2784 & MUC & \\
\hline ARK-XXI/2 & PS68/275-3 & S3 & $25 / 08 / 2005$ & $78^{\circ} 36.59^{\prime}$ & $5^{\circ} 04.20^{\prime}$ & 2339 & MUC & \\
\hline MSM2/4 & MSM2/780-4 & HG-IV & $24 / 08 / 2006$ & $79^{\circ} 03.93^{\prime}$ & $4^{\circ} 10.84^{\prime}$ & 2411 & MUC & \\
\hline MSM2/4 & MSM2/803-2 & S3 & $27 / 08 / 2006$ & $78^{\circ} 36.40^{\prime}$ & $5^{\circ} 04.12^{\prime}$ & 2293 & MUC & \\
\hline MSM2/4 & MSM2/864-1 & N3 & 04/09/2006 & $79^{\circ} 36.24^{\prime}$ & $5^{\circ} 16.31^{\prime}$ & 2650 & MUC & \\
\hline ARK-XXII/2 & PS70/147-1 & HG-IV & $10 / 07 / 2007$ & $79^{\circ} 03.92^{\prime}$ & $4^{\circ} 10.55^{\prime}$ & 2477 & MUC & \\
\hline ARK-XXII/2 & PS70/164-1 & S3 & $12 / 07 / 2007$ & $78^{\circ} 36.98^{\prime}$ & $5^{\circ} 00.38^{\prime}$ & 2374 & OFOS (start) & \\
\hline ARK-XXII/2 & PS70/164-1 & $\mathrm{S} 3$ & $12 / 07 / 2007$ & $78^{\circ} 37.00^{\prime}$ & $5^{\circ} 05.96^{\prime}$ & 2351 & OFOS (end) & 219 \\
\hline ARK-XXII/2 & PS70/170-1 & HG-IV & $12 / 07 / 2007$ & $79^{\circ} 02.27^{\prime}$ & $4^{\circ} 11.44^{\prime}$ & 2613 & OFOS (start) & \\
\hline ARK-XXII/2 & PS70/170-1 & HG-IV & $12 / 07 / 2007$ & $79^{\circ} 03.89^{\prime}$ & $4^{\circ} 17.22^{\prime}$ & 2422 & OFOS (end) & 186 \\
\hline ARK-XXII/2 & PS70/174-1 & S3 & $13 / 07 / 2007$ & $78^{\circ} 36.54^{\prime}$ & $5^{\circ} 03.82^{\prime}$ & 2354 & MUC & \\
\hline ARK-XXII/2 & PS70/197-1 & N3 & $17 / 07 / 2007$ & $79^{\circ} 36.32^{\prime}$ & $5^{\circ} 09.23$ & 2804 & MUC & \\
\hline ARK-XXII/2 & PS70/202-1 & N3 & $17 / 07 / 2007$ & $79^{\circ} 35.82^{\prime}$ & $5^{\circ} 10.02^{\prime}$ & 2800 & OFOS (start) & \\
\hline ARK-XXII/2 & PS70/202-1 & N3 & $17 / 07 / 2007$ & $79^{\circ} 34.10^{\prime}$ & $5^{\circ} 14.83^{\prime}$ & 2681 & OFOS (end) & 220 \\
\hline ARK-XXIII/2 & PS72/122-2 & HG-IV & 09/07/2008 & $79^{\circ} 03.83^{\prime}$ & $4^{\circ} 10.61^{\prime}$ & 2473 & MUC & \\
\hline ARK-XXIII/2 & PS72/129-3 & S3 & $10 / 07 / 2008$ & $78^{\circ} 36.48^{\prime}$ & $5^{\circ} 03.68^{\prime}$ & 2343 & MUC & \\
\hline ARK-XXIII/2 & PS72/146-1 & N3 & $14 / 07 / 2008$ & $79^{\circ} 35.75^{\prime}$ & $5^{\circ} 10.91^{\prime}$ & 2781 & MUC & \\
\hline ARK-XXIII/2 & PS72/156-1 & HG-IV & $16 / 07 / 2008$ & $79^{\circ} 00.25^{\prime}$ & $3^{\circ} 30.71^{\prime}$ & 2751 & AGT & \\
\hline ARK-XXIV/2 & PS74/118-2 & N3 & 16/07/2009 & $79^{\circ} 36.24^{\prime}$ & $5^{\circ} 10.07^{\prime}$ & 2787 & MUC & \\
\hline ARK-XXIV/2 & PS74/121-1 & HG-IV & 16/07/2009 & $79^{\circ} 03.89^{\prime}$ & $4^{\circ} 10.92^{\prime}$ & 2464 & MUC & \\
\hline ARK-XXIV/2 & PS74/129-3 & S3 & 18/07/2009 & $78^{\circ} 36.48^{\prime}$ & $5^{\circ} 04.38^{\prime}$ & 2340 & MUC & \\
\hline ARK-XXV/2 & PS76/124-4 & S3 & 04/07/2010 & $78^{\circ} 36.37^{\prime}$ & $5^{\circ} 03.97^{\prime}$ & 2341 & MUC & \\
\hline ARK-XXV/2 & PS76/142-3 & HG-IV & 08/07/2010 & $79^{\circ} 03.87^{\prime}$ & $4^{\circ} 10.38^{\prime}$ & 2461 & MUC & \\
\hline ARK-XXV/2 & PS76/181-2 & N3 & $15 / 07 / 2010$ & $79^{\circ} 35.69^{\prime}$ & $5^{\circ} 13.24^{\prime}$ & 2768 & MUC & \\
\hline ARK-VI/2 & PS78/143-2 & HG-IV & $16 / 07 / 2011$ & $79^{\circ} 01.82^{\prime}$ & $4^{\circ} 09.47^{\prime}$ & 2637 & OFOS (start) & \\
\hline ARK-VI/2 & PS78/143-2 & HG-IV & 16/07/2011 & $79^{\circ} 03.90^{\prime}$ & $4^{\circ} 17.19^{\prime}$ & 2407 & OFOS (end) & 178 \\
\hline ARK-VI/2 & PS78/143-7 & HG-IV & 17/07/2011 & $79^{\circ} 03.86^{\prime}$ & $4^{\circ} 10.58^{\prime}$ & 2468 & MUC & \\
\hline ARK-VI/2 & PS78/171-1 & N3 & 27/07/2011 & $79^{\circ} 35.84^{\prime}$ & $5^{\circ} 09.95$ & 2788 & OFOS (start) & \\
\hline ARK-VI/2 & PS78/171-1 & N3 & $27 / 07 / 2011$ & $79^{\circ} 34.11^{\prime}$ & $5^{\circ} 15.08^{\prime}$ & 2663 & OFOS (end) & 209 \\
\hline ARK-VI/2 & PS78/171-6 & N3 & $27 / 07 / 2011$ & $79^{\circ} 35.71^{\prime}$ & $5^{\circ} 13.26^{\prime}$ & 2753 & MUC & \\
\hline ARK-VI/2 & PS78/182-1 & $\mathrm{S} 3$ & $30 / 07 / 2011$ & $78^{\circ} 37.00^{\prime}$ & $5^{\circ} 00.19^{\prime}$ & 2366 & OFOS (start) & \\
\hline ARK-VI/2 & PS78/182-1 & S3 & $30 / 07 / 2011$ & $78^{\circ} 36.99^{\prime}$ & $5^{\circ} 09.95^{\prime}$ & 2351 & OFOS (end) & 205 \\
\hline ARK-VI/2 & PS78/182-3 & S3 & $30 / 07 / 2011$ & $78^{\circ} 36.38^{\prime}$ & $5^{\circ} 03.92^{\prime}$ & 2341 & MUC & \\
\hline ARK-XXVII/2 & PS80/165-8 & HG-IV & $16 / 07 / 2012$ & $79^{\circ} 03.86^{\prime}$ & $4^{\circ} 10.85^{\prime}$ & 2467 & MUC & \\
\hline ARK-XXVII/2 & PS80/176-1 & S3 & 19/07/2012 & $78^{\circ} 37.04^{\prime}$ & $5^{\circ} 00.07^{\prime}$ & 2361 & OFOS (start) & \\
\hline ARK-XXVII/2 & PS80/176-1 & S3 & $20 / 07 / 2012$ & $78^{\circ} 37.00^{\prime}$ & $5^{\circ} 08.56^{\prime}$ & 2352 & OFOS (end) & 198 \\
\hline ARK-XXVII/2 & PS80/176-7 & S3 & $20 / 07 / 2012$ & $78^{\circ} 36.59^{\prime}$ & $5^{\circ} 03.96^{\prime}$ & 2340 & MUC & \\
\hline ARK-XXVII/2 & PS80/179-3 & HG-IV & $21 / 07 / 2012$ & $79^{\circ} 01.98^{\prime}$ & $4^{\circ} 09.75^{\prime}$ & 2630 & OFOS (start) & \\
\hline
\end{tabular}


Table 1 (continued)

\begin{tabular}{|c|c|c|c|c|c|c|c|c|}
\hline Cruise & $\begin{array}{l}\text { Station } \\
\text { number }\end{array}$ & $\begin{array}{c}\text { Station } \\
\text { name }\end{array}$ & $\begin{array}{c}\text { Date } \\
\text { (dd/mm/yr) }\end{array}$ & $\begin{array}{l}\text { Latitude } \\
\text { (N) }\end{array}$ & $\begin{array}{l}\text { Longitude } \\
\text { (E) }\end{array}$ & $\begin{array}{l}\text { Depth } \\
\text { (m) }\end{array}$ & Gear & $\begin{array}{c}\text { No. of images } \\
\text { analysed }\end{array}$ \\
\hline ARK-XXVII/2 & PS80/179-3 & HG-IV & $21 / 07 / 2012$ & $79^{\circ} 03.88^{\prime}$ & $4^{\circ} 09.75^{\prime}$ & 2409 & OFOS (end) & 219 \\
\hline ARK-XXVII/2 & PS80/188-2 & N3 & $25 / 07 / 2012$ & $79^{\circ} 36.23^{\prime}$ & $5^{\circ} 10.23^{\prime}$ & 2742 & MUC & \\
\hline ARK-XXVII/2 & PS80/193-1 & N3 & $26 / 07 / 2012$ & $79^{\circ} 36.04^{\prime}$ & $5^{\circ} 09.88^{\prime}$ & 2748 & OFOS (start) & \\
\hline ARK-XXVII/2 & PS80/193-1 & N3 & $26 / 07 / 2012$ & $79^{\circ} 33.53^{\prime}$ & $5^{\circ} 16.99^{\prime}$ & 2609 & OFOS (end) & 219 \\
\hline MSM29 & MSM29/431-3 & N3 & $27 / 06 / 2013$ & $79^{\circ} 35.71^{\prime}$ & $5^{\circ} 12.57^{\prime}$ & 2722 & MUC & \\
\hline MSM29 & MSM29/439-3 & S3 & $01 / 07 / 2013$ & $78^{\circ} 37.20^{\prime}$ & $5^{\circ} 01.03^{\prime}$ & 2318 & MUC & \\
\hline MSM29 & MSM29/440-1 & S3 & $02 / 07 / 2013$ & $78^{\circ} 37.04^{\prime}$ & $5^{\circ} 00.08^{\prime}$ & 2316 & OFOS (start) & \\
\hline MSM29 & MSM29/440-1 & S3 & 02/07/2013 & $78^{\circ} 37.00^{\prime}$ & $5^{\circ} 08.58^{\prime}$ & 2304 & OFOS (end) & 219 \\
\hline MSM29 & MSM29/445-1 & N3 & 05/07/2013 & $79^{\circ} 35.98^{\prime}$ & $5^{\circ} 09.62^{\prime}$ & 2747 & OFOS (start) & \\
\hline MSM29 & MSM29/445-1 & N3 & $05 / 07 / 2013$ & $79^{\circ} 34.79^{\prime}$ & $5^{\circ} 13.13^{\prime}$ & 2645 & OFOS (end) & 220 \\
\hline MSM29 & MSM29/453-9 & HG-IV & 07/09/2013 & $79^{\circ} 04.82^{\prime}$ & $4^{\circ} 04.71^{\prime}$ & 2464 & MUC & \\
\hline ARK-XXVIII/1 & PS85/460-4 & HG-IV & $22 / 06 / 2014$ & $79^{\circ} 03.91^{\prime}$ & $4^{\circ} 10.98^{\prime}$ & 2403 & MUC & \\
\hline ARK-XXVIII/1 & PS85/461-1 & HG-IV & $24 / 06 / 2014$ & $79^{\circ} 02.01^{\prime}$ & $4^{\circ} 09.98^{\prime}$ & 2566 & OFOS (start) & \\
\hline ARK-XXVIII/1 & PS85/461-1 & HG-IV & $24 / 06 / 2014$ & $79^{\circ} 03.89^{\prime}$ & $4^{\circ} 17.26^{\prime}$ & 2353 & OFOS (end) & 220 \\
\hline ARK-XXVIII/1 & PS85/474-1 & N3 & $26 / 06 / 2014$ & $79^{\circ} 35.92^{\prime}$ & $5^{\circ} 10.15^{\prime}$ & 2721 & OFOS (start) & \\
\hline ARK-XXVIII/1 & PS85/474-1 & N3 & $26 / 06 / 2014$ & $79^{\circ} 34.13^{\prime}$ & $5^{\circ} 15.29^{\prime}$ & 2600 & OFOS (end) & 218 \\
\hline ARK-XXIX/2 & PS93/048-8 & S3 & $24 / 07 / 2015$ & $78^{\circ} 37.02^{\prime}$ & $5^{\circ} 09.56^{\prime}$ & 2351 & OFOS (start) & \\
\hline ARK-XXIX/2 & PS93/048-8 & S3 & $24 / 07 / 2015$ & $78^{\circ} 36.98^{\prime}$ & $4^{\circ} 59.39^{\prime}$ & 2367 & OFOS (end) & 220 \\
\hline ARK-XXIX/2 & PS93/048-11 & S3 & $25 / 07 / 2015$ & $78^{\circ} 35.98^{\prime}$ & $5^{\circ} 04.07^{\prime}$ & 2342 & MUC & \\
\hline ARK-XXIX/2 & PS93/050-11 & HG-IV & $27 / 07 / 2015$ & $79^{\circ} 02.02^{\prime}$ & $4^{\circ} 09.91^{\prime}$ & 2629 & OFOS (start) & \\
\hline ARK-XXIX/2 & PS93/050-11 & HG-IV & $27 / 07 / 2015$ & $79^{\circ} 03.93^{\prime}$ & $4^{\circ} 17.28^{\prime}$ & 2406 & OFOS (end) & 220 \\
\hline ARK-XXIX/2 & PS93/050-19 & HG-IV & $27 / 07 / 2015$ & $79^{\circ} 03.91^{\prime}$ & $4^{\circ} 10.79^{\prime}$ & 2465 & MUC & \\
\hline ARK-XXIX/2 & PS93/062-1 & N3 & 03/08/2015 & $79^{\circ} 35.92^{\prime}$ & $5^{\circ} 10.18^{\prime}$ & 2787 & OFOS (start) & \\
\hline ARK-XXIX/2 & PS93/062-1 & N3 & $03 / 08 / 2015$ & $79^{\circ} 34.15^{\prime}$ & $5^{\circ} 15.36^{\prime}$ & 2658 & OFOS (end) & 192 \\
\hline ARK-XXIX/2 & PS93/085-2 & N3 & $11 / 08 / 2015$ & $79^{\circ} 36.25^{\prime}$ & $5^{\circ} 10.28^{\prime}$ & 2783 & MUC & \\
\hline
\end{tabular}

Specimens of Kolga hyalina, Elpidia heckeri and Mohnia spp. were obtained during expeditions ARKXX/1a (2004), ARK-XXI/2 (2005) and ARK-XXIII/2 (2008) of RV 'Polarstern' with a 3-m Agassiz trawl (AGT), which was towed along the seafloor for $30 \mathrm{~min}$ (see Bergmann et al. 2009). All samples were fixed in $4 \%$ formalin, washed and then preserved in $70 \%$ ethanol. Table 1 summarises all gear deployments.

\section{Image selection and analysis}

The first 220 automatically triggered images of each transect of each year were selected for the study. If 220 images were not available in the section to be analysed because of images being unsuitable due to inappropriate lighting, blurriness or presence of sediment clouds, then as many as possible were used instead.

The images were analysed in the web-2.0 based platform BIIGLE 2.0 (Benthic Image Indexing and Graphical Labelling Environment) (Langenkämper et al. 2017). Each image was labelled by a taxonomic expert at a high zoom and in a shaded room to reduce error introduced due to external glare. Kolga hyalina, Elpidia heckeri and Mohnia spp. were all measured 'tip to tip', giving the length of the entire animal (Fig. 2). This was combined with the 3 laser points (2 in 2007), which were used as a standard to calculate the area of each image to allow for length measurement.

\section{Measurement of sampled specimens and conversions}

Measurements of the sampled specimens of all 3 taxa were also taken at the longest 'tip to tip'. Length measurements were taken using a digital calliper.

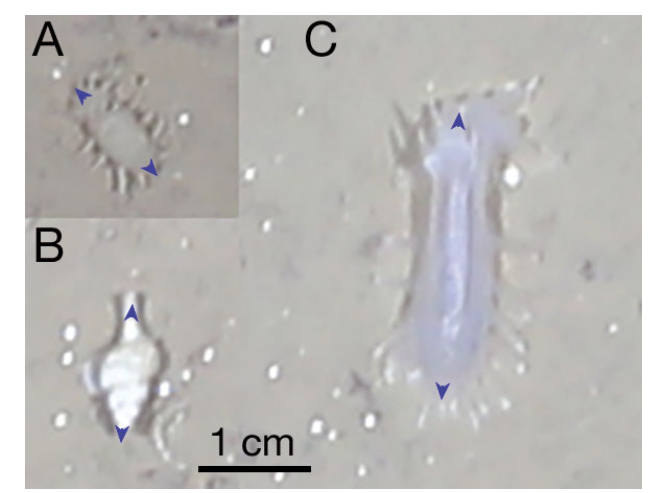

Fig. 2. Examples of (A) Elpidia heckeri, (B) Mohnia spp. and (C) Kolga hyalina. Blue arrows indicate axis of measurement 
Wet-weight measurements of intact organisms were taken at an accuracy of 3 decimal places as blotted wet weights. Mohnia spp. samples included specimens of the congeners Mohnia mohni and Mohnia danielsseni, which had previously been identified and separated by A. Warén (Swedish Museum of Natural History).

Conversion factors to estimate the weight of individuals based on the relationship with length for all 3 organisms in images are given in the form:

$$
W=A \times L^{B}
$$

where $W$ is the preserved wet weight $(g), L$ is the organism length (mm) and $A$ and $B$ are coefficients derived from the linear regression of the log-converted formula above:

$$
\log W=\log A+(B \times \log L)
$$

An adjusted $\mathrm{R}^{2}$ value was calculated for each conversion factor to evaluate the goodness of fit.

\section{Data analysis}

The megafaunal abundance for each image was extracted from BIIGLE and converted to density (number of ind. $\mathrm{m}^{-2}$ ). Organism length $(\mathrm{mm})$ for each individual labelled in the study was also extracted from BIIGLE and then converted to mass of the individual $(\mathrm{g}$ ) using the derived conversion factors. Standard parametric tests (Minitab 17: 1-way ANOVA with Tukey comparisons) were used to compare environmental sediment parameters and to compare mean mass per individual with abundance. Non-parametric Kruskal-Wallis tests followed by pairwise Mann-Whitney $U$-tests were applied, using a Bonferroni correction (N3; p = 0.05/21 $=0.0024$, HG-IV; $\mathrm{p}=0.05 / 15=0.0033, \mathrm{~S} 3 ; \mathrm{p}=$ $0.05 / 15=0.0033$ ) to megafaunal abundance and individual/overall biomass, as the variance of the data was not homogenous. To test correlation between population density and mean mass per individual Spearman's rank correlation tests were used.

\section{RESULTS}

\section{Conversion factors}

Using sampled specimens we were able to ascertain the length to weight conversions for Kolga hyalina, Elpidia heckeri and Mohnia spp. (Table 2). It should be noted that the 2 Mohnia congeners, Mohnia mohni and Mohnia danielsseni cannot be distinguished via image analysis and therefore had to be pooled. Of the physical individuals sampled $(\mathrm{N}=91)$, 11 belonged to $M$. danielsseni and 80 belonged to $M$. mohni with the conversion factor producing an adjusted $\mathrm{R}^{2}$ of 0.862 .

Table 3. Mean mass per individual (g) and total number of individuals measured (in parentheses) of Kolga hyalina, Elpidia heckeri and Mohnia spp. recorded from photographic transects conducted at HAUSGARTEN stations N3, HG-IV and S3 between 2004 and 2015. SE: standard error of the mean; K-W: Kruskal Wallis test; M-W: Mann-Whitney $U$-test. Dashes (-):

\begin{tabular}{|c|c|c|c|c|c|c|c|c|c|}
\hline Taxon & Station & 2004 & $\pm \mathrm{SE}$ & 2007 & $\pm \mathrm{SE}$ & 2011 & $\pm \mathrm{SE}$ & 2012 & $\pm \mathrm{SE}$ \\
\hline \multirow{3}{*}{$\begin{array}{l}\text { Kolga } \\
\text { hyalina }\end{array}$} & N3 & - & & 3.004 (4459) & 0.014 & 1.559 (3558) & 0.008 & $1.807(3276)$ & 0.009 \\
\hline & HG-IV & $6.116(169)$ & 0.149 & - & & 3.138 (142) & 0.082 & 2.935 (219) & 0.065 \\
\hline & S3 & - & & - & & - & & - & \\
\hline \multirow{3}{*}{$\begin{array}{l}\text { Elpidia } \\
\text { heckeri }\end{array}$} & N3 & $0.424(42)$ & 0.042 & $0.236(364)$ & 0.005 & $0.156(604)$ & 0.003 & $0.166(601)$ & 0.004 \\
\hline & HG-IV & $0.180(281)$ & 0.006 & $0.211(130)$ & 0.012 & $0.113(231)$ & 0.004 & $0.087(778)$ & 0.002 \\
\hline & S3 & $0.278(89)$ & 0.014 & $0.197(77)$ & 0.019 & $0.192(380)$ & 0.005 & $0.139(400)$ & 0.004 \\
\hline \multirow{3}{*}{$\begin{array}{l}\text { Mohnia } \\
\text { spp. }\end{array}$} & N3 & $0.616(275)$ & 0.022 & $0.377(1846)$ & 0.008 & $0.208(1708)$ & 0.005 & $0.287(1782)$ & 0.041 \\
\hline & HG-IV & $0.323(255)$ & 0.018 & $0.453(240)$ & 0.021 & $0.246(458)$ & 0.011 & $0.254(1098)$ & 0.006 \\
\hline & S3 & $0.401(330)$ & 0.014 & $0.367(224)$ & 0.015 & $0.424(267)$ & 0.015 & $0.298(439)$ & 0.011 \\
\hline
\end{tabular}
taxon was not found; ns: years where no sampling was possible 


\section{Biomass and density of Kolga hyalina over time}

Significant temporal differences were observed in the mean $( \pm \mathrm{SE})$ weight of individuals (hereafter referred to solely as mass) for Kolga hyalina at N3 (KW, M-W, p < 0.0005, $\chi^{2}=6917.10, \mathrm{df}=6$ ). Kolga were initially absent with the individuals of largest mass being seen in $2007\left(3.004 \pm 0.014 \mathrm{~g}_{\text {ind. }}{ }^{-1}\right)$, the year in which they were first observed, followed by a decrease before increasing again in 2015 (2.633 \pm $0.025 \mathrm{~g}$ ind. $^{-1}$ ) (Table 3). A similar overall trend was seen at HG-IV (K-W, M-W, p $<0.0005, \chi^{2}=273.64$, df $=5)$. However, mean mass per individual went from the highest observed in $2004\left(6.116 \pm 0.149 \mathrm{~g} \mathrm{ind}^{-1}\right)$ to complete absence in 2007. After this point, Kolga returned with an increased mass observed in 2015 $\left(4.123 \pm 0.321 \mathrm{~g} \mathrm{ind}^{-1}\right)$.

Kolga showed significant temporal differences in density at N3 (K-W, M-W, p < 0.0005, $\chi^{2}=1059.67$, df $=6$ ), decreasing in each observed year from 2004 to 2015. Significant temporal variations in density were also seen at HG-IV (K-W, M-W, p $<0.0005, \chi^{2}$ $=140.83, \mathrm{df}=5)$. Densities were highest in 2004 and $2012\left(0.271 \pm 0.037\right.$ and $0.289 \pm 0.021$ ind. $\mathrm{m}^{-2}$, respectively), with the lowest density in 2015 (0.027 \pm 0.007 ind. $\mathrm{m}^{-2}$ ). When comparing the mean mass per individual to the density of Kolga, we see that from 2011 (N3) and 2012 (HG-IV) the overall trend is that as density decreases the size of the individual increases (Figs. $3 \& 4$ ) (Spearman's, $p=0.014$, rho $=$ $-0.857)$. In the years before, the trends in biomass and density followed the same patterns.

The overall biomass of Kolga showed significant temporal variation at N3 $\left(\mathrm{K}-\mathrm{W}, \mathrm{M}-\mathrm{W}, \mathrm{p}<0.0005, \chi^{2}=\right.$ 1052.63, df $=6)$ and HG-IV (K-W, M-W, p<0.0005, $\chi^{2}$ $=135.81, \mathrm{df}=5$ ). Both stations showed an overall declining trend in biomass, except during 2004 at N3, when Kolga was absent, ranging from $14.313 \pm 0.253$ (2007) to $3.541 \pm 0.099 \mathrm{~g} \mathrm{~m}^{-2}$ (2014) and $1.660 \pm 0.229$
(2004) to $0.110 \pm 0.029 \mathrm{~g} \mathrm{~m}^{-2}$ (2015), at N3 and HG-IV, respectively (Table 4, Fig. 5). The overall biomass of Kolga was higher at N3 from 2007 onwards than at HG-IV.

\section{Biomass and density of Elpidia heckeri over time}

Elpidia heckeri showed significant temporal variations in mass per individual at all 3 stations $(\mathrm{N} 3: \mathrm{K}-\mathrm{W}$, $\mathrm{M}-\mathrm{W}, \mathrm{p}<0.0005, \chi^{2}=312.70, \mathrm{df}=6$; HG-IV: K-W, M$\mathrm{W}, \mathrm{p}<0.0005, \chi^{2}=513.64, \mathrm{df}=5$; S3: K-W, M-W, $\mathrm{p}<$ $0.0005, \chi^{2}=714.69, \mathrm{df}=5$ ) (Table 3). Overall, there was a decreasing trend between $2004(0.424 \pm 0.042$ $\mathrm{g}$ ind. $\left.{ }^{-1}\right)$ and $2011\left(0.156 \pm 0.003 \mathrm{~g} \mathrm{ind}^{-1}\right)$ at N3 and $2004\left(0.401 \pm 0.014 \mathrm{~g} \mathrm{ind}^{-1}\right)$ and $2013(0.077 \pm 0.002$ $\mathrm{g}$ ind. $\left.{ }^{-1}\right)$ at $\mathrm{S} 3$ before levelling off. A decreasing trend was also seen between $2007\left(0.211 \pm 0.012 \mathrm{~g} \mathrm{ind}^{-1}\right)$ and $2012\left(0.087 \pm 0.002 \mathrm{~g} \mathrm{ind}^{-1}\right)$ at HG-IV, however, after 2012 mean mass per individual increased to $2015\left(0.160 \pm 0.003 \mathrm{~g} \mathrm{ind}^{-1}\right)$ (Table 3, Figs. 3 \& 4).

Significant temporal variations were also observed in density at all 3 stations (N3: K-W, M-W, p $<0.0005$, $\chi^{2}=522.33, \mathrm{df}=6$; HG-IV: $\mathrm{K}-\mathrm{W}, \mathrm{M}-\mathrm{W}, \mathrm{p}<0.0005, \chi^{2}=$ 747.89, df $=5$; S3: K-W, M-W, p < 0.0005, $\chi^{2}=674.77$, $\mathrm{df}=5$ ) (Table 5). Abundances increased at all 3 stations, with a 9-fold, 4 -fold and 10-fold increase at N3, HG-IV and S3, respectively, from 2004-2015 (Fig. 3). When density increases, the mean mass per individual decreases (Spearman's, $p=0.002$, rho $=-0.654$ ). The exception to this is at HG-IV, from 2012-2015, where both increased in parallel.

The overall biomass of Elpidia was subject to significant temporal variation at all stations (N3: K-W, M-W, p < 0.0005, $\chi^{2}=396.20, \mathrm{df}=6$; HG-IV: K-W, M$\mathrm{W}, \mathrm{p}<0.0005, \chi^{2}=751.90, \mathrm{df}=6$; S3: K-W, M-W, $\mathrm{p}<$ $0.0005, \chi^{2}=561.53, \mathrm{df}=6$ ) (Table 4, Fig. 5). The main causes for differences are the increases in 2015 at N3 and S3 and in 2014 and 2015 at HG-IV.

Table 3 (continued)

\begin{tabular}{ccccccccc}
\hline 2013 & $\pm \mathrm{SE}$ & 2014 & $\pm \mathrm{SE}$ & 2015 & $\pm \mathrm{SE}$ & Test used & $\mathrm{p}$ & $\chi^{2}$ \\
\hline $2.172(2053)$ & 0.013 & $1.744(1827)$ & 0.012 & $2.633(892)$ & 0.025 & $\mathrm{~K}-\mathrm{W}, \mathrm{M}-\mathrm{W}$ & $<0.0005$ & 6917.10 \\
ns & & $2.863(35)$ & 0.240 & $4.123(19)$ & 0.321 & $\mathrm{~K}-\mathrm{W}, \mathrm{M}-\mathrm{W}$ & $<0.0005$ & 273.64 \\
- & & $\mathrm{ns}$ & & - & & & \\
$0.164(540)$ & 0.004 & $0.155(732)$ & 0.003 & $0.191(802)$ & 0.003 & $\mathrm{~K}-\mathrm{W}, \mathrm{M}-\mathrm{W}$ & $<0.0005$ & 312.70 \\
ns & & $0.142(1437)$ & 0.002 & $0.160(1321)$ & 0.003 & $\mathrm{~K}-\mathrm{W}, \mathrm{M}-\mathrm{W}$ & $<0.0005$ & 513.64 \\
$0.077(541)$ & 0.002 & $\mathrm{~ns}$ & & $0.111(1816)$ & 0.002 & $\mathrm{~K}-\mathrm{W}, \mathrm{M}-\mathrm{W}$ & $<0.0005$ & 714.49 \\
$0.303(1360)$ & 0.007 & $0.299(1546)$ & 0.006 & $0.371(705)$ & 0.010 & $\mathrm{~K}-\mathrm{W}, \mathrm{M}-\mathrm{W}$ & $<0.0005$ & 1194.94 \\
ns & & $0.244(921)$ & 0.007 & $0.316(416)$ & 0.012 & $\mathrm{~K}-\mathrm{W}, \mathrm{M}-\mathrm{W}$ & $<0.0005$ & 216.76 \\
$0.636(443)$ & 0.028 & $\mathrm{~ns}$ & & $0.360(542)$ & 0.015 & $\mathrm{~K}-\mathrm{W}, \mathrm{M}-\mathrm{W}$ & $<0.0005$ & 193.77 \\
\hline
\end{tabular}




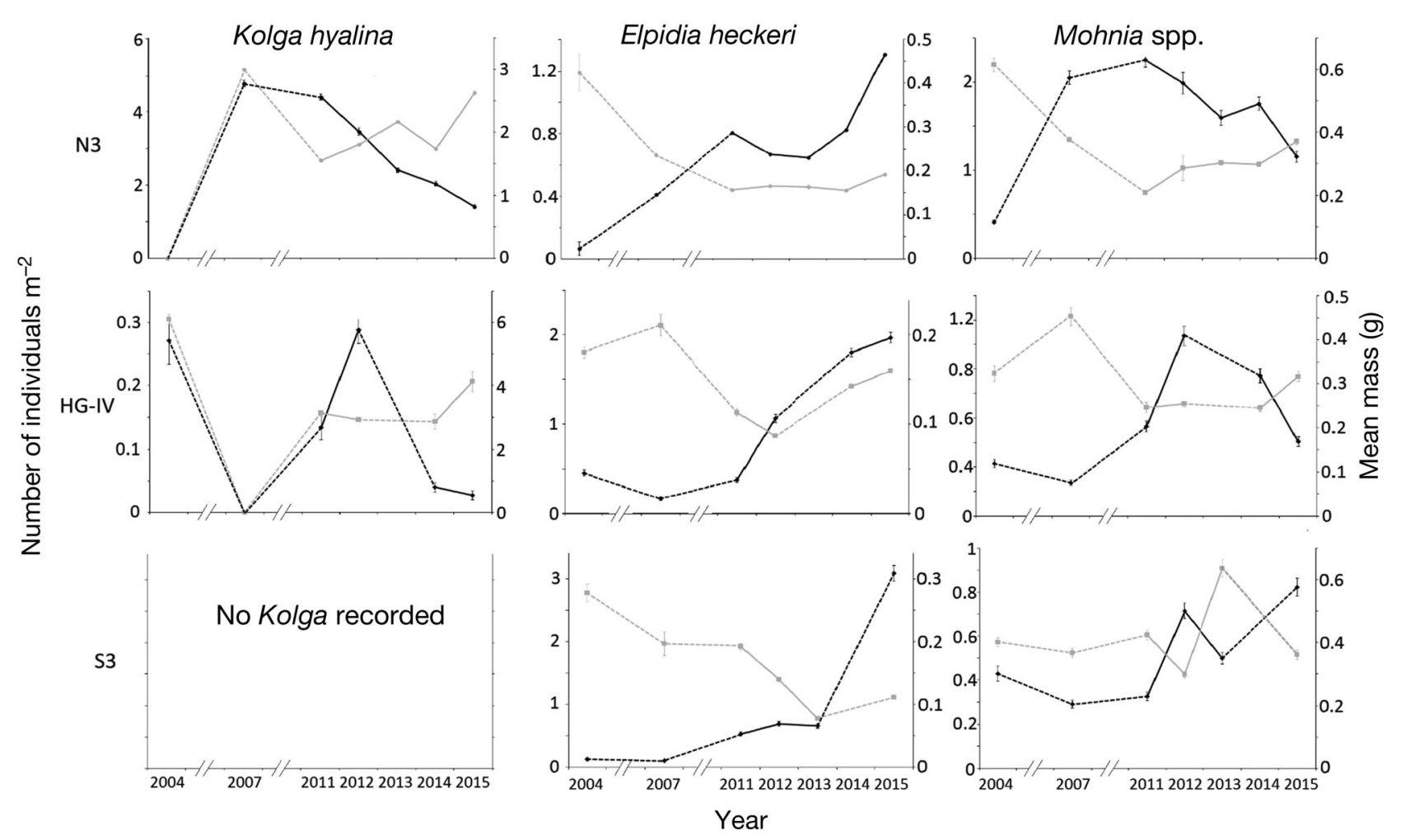

Fig. 3. Mean densities of organisms (ind. $\mathrm{m}^{-2}$; black line) and mean mass per individual (g; grey line) of Kolga hyalina, Elpidia heckeri and Mohnia spp. recorded from photographic transects taken at HAUSGARTEN stations N3, HG-IV and S3 for each year. Dashed lines link years between which sampling was not possible. Error bars represent the standard error of the mean

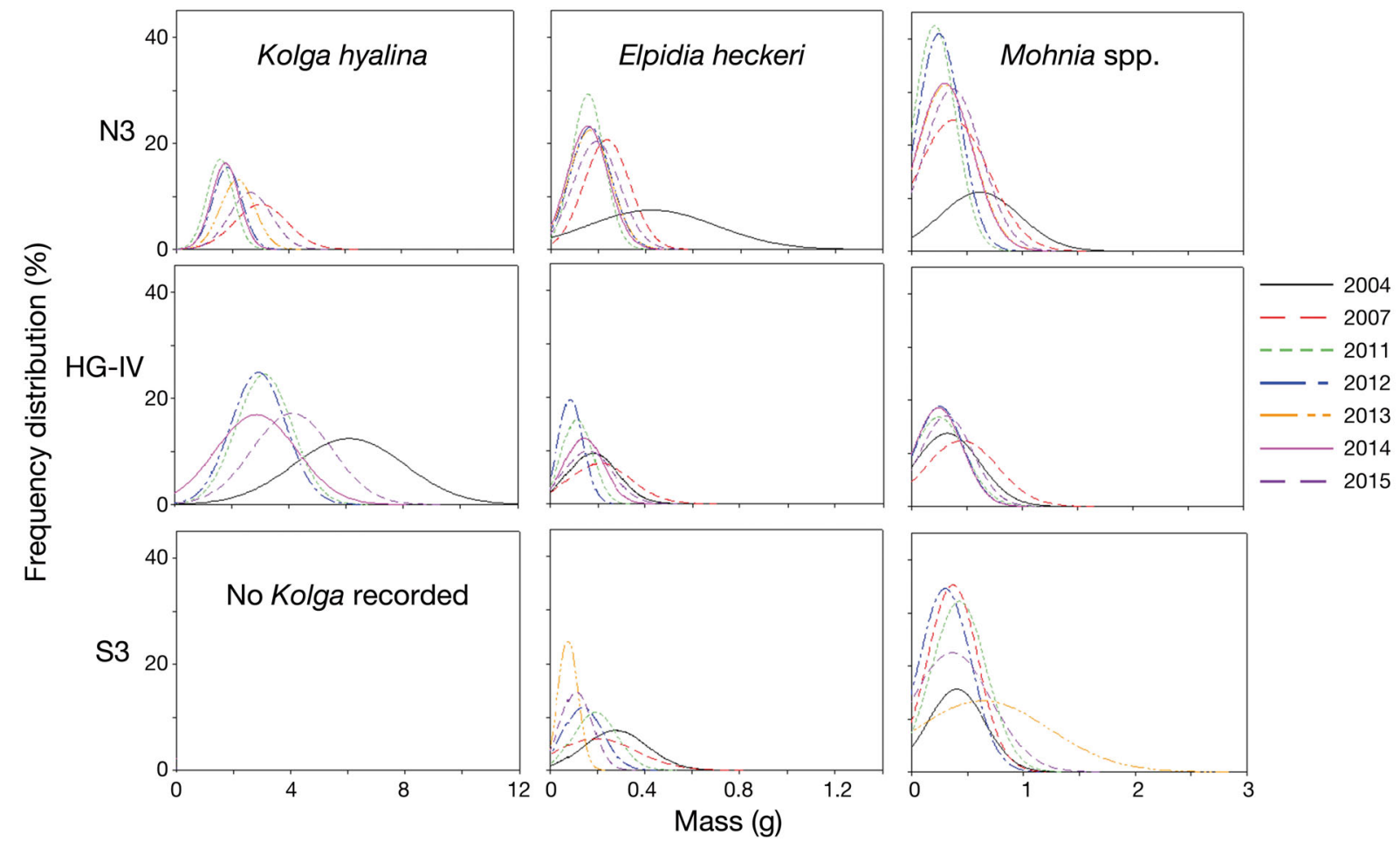

Fig. 4. Percentage distribution of mass of individuals (g) of Kolga hyalina, Elpidia heckeri and Mohnia spp. recorded from photographic transects taken at HAUSGARTEN stations N3, HG-IV and S3 for each year 


\section{Biomass and density of Mohnia spp. over time}

The mean mass per individual of Mohnia spp. showed significant temporal variation at all 3 stations (N3: K-W, M-W, $\mathrm{p}<0.0005, \chi^{2}=1194.94, \mathrm{df}=6$; HGIV: $\mathrm{K}-\mathrm{W}, \mathrm{M}-\mathrm{W}, \mathrm{p}<0.0005, \chi^{2}=216.76, \mathrm{df}=5$; S3: K-W, $\mathrm{M}-\mathrm{W}, \mathrm{p}<0.0005, \chi^{2}=193.77, \mathrm{df}=5$ ) (Fig. 3). Between $2004\left(0.616 \pm 0.022 \mathrm{~g}\right.$ ind.$\left.^{-1}\right)$ and $2011(0.208 \pm 0.005 \mathrm{~g}$ ind. ${ }^{-1}$ ) the mean mass per individual at N3 decreased followed by an overall increasing trend $(2015 ; 0.371 \pm$ $0.010 \mathrm{~g} \mathrm{ind}^{-1}$ ). The mean mass per individual at HGIV peaked in $2007\left(0.453 \pm 0.021 \mathrm{~g}\right.$ ind..$\left.^{-1}\right)$, decreased in $2011\left(0.246 \pm 0.001 \mathrm{~g} \mathrm{ind.}^{-1}\right)$, then levelled off before a slight increase in $2015\left(0.316 \pm 0.012 \mathrm{~g} \mathrm{ind}^{-1}\right)$. At S3, it was at a stable level until a decrease in 2012 $\left(0.298 \pm 0.011 \mathrm{~g}\right.$ ind.$\left.^{-1}\right)$ followed by a maximum in $2013\left(0.636 \pm 0.028 \mathrm{~g} \mathrm{ind}^{-1}\right)$, before returning to original levels in $2015\left(0.360 \pm 0.015 \mathrm{~g}^{\text {ind. }}{ }^{-1}\right)$ (Table 3$)$.

Densities of Mohnia spp. also showed significant temporal variations at all 3 stations (N3: K-W, M-W, $\mathrm{p}<$ 0.0005, $\chi^{2}=482.33$, df $=6$; HG-IV: K-W, M-W, $\mathrm{p}<$ $0.0005, \chi^{2}=367.56, \mathrm{df}=5$; S3: K-W, M-W, $\mathrm{p}<0.0005, \chi^{2}$ $=199.28, \mathrm{df}=5$ ) (Fig. 3). Overall when an increase in mass was observed the density was lowered and vice versa (Spearman's, $p=0.004$, rho $=-0.626$ ) (Table 5).

The overall biomass of Mohnia each year showed significant temporal variation at all 3 stations (N3: K$\mathrm{W}, \mathrm{M}-\mathrm{W}, \mathrm{p}<0.0005, \chi^{2}=266.93, \mathrm{df}=6$; HG-IV: K-W, $\mathrm{M}-\mathrm{W}, \mathrm{p}<0.0005, \chi^{2}=228.27, \mathrm{df}=5$; S3: K-W, M-W, $\mathrm{p}<0.0005, \chi^{2}=201.86, \mathrm{df}=5$ ) (Fig. 5). Overall, HGIV and S3 showed similar total biomass of Mohnia with the largest difference being an increase at HGIV between $2011\left(0.180 \pm 0.011 \mathrm{~g} \mathrm{~m}^{-2}\right)$ and 2012 $\left(0.374 \pm 0.019 \mathrm{~g} \mathrm{~m}^{-2}\right)$. On the whole, overall biomass was higher at N3, with a large increase between $2004\left(0.254 \pm 0.012 \mathrm{~g} \mathrm{~m}^{-2}\right)$ and $2007(0.773 \pm 0.029 \mathrm{~g}$ $\left.\mathrm{m}^{-2}\right)$, before decreasing in $2011\left(0.469 \pm 0.018 \mathrm{~g} \mathrm{~m}^{-2}\right)$ and levelling off (Table 4).

\section{Temporal variations in biogeochemical sediment parameters}

There were significant variations in phospholipid concentrations and phytodetrital matter availability (CPE) at N3 and S3, with particularly elevated levels of CPE from 2007 onwards (Fig. 6), while there was no significant variation in protein concentrations or particulate organic matter (data not shown). Results from HG-IV mirror these results. Significant variations in CPE (ANOVA, $\mathrm{p}<0.0005, F=7.85$, df $=13$ ) followed the trend of the other stations, with a sustained increase from 2007 onwards. Significant vari- 


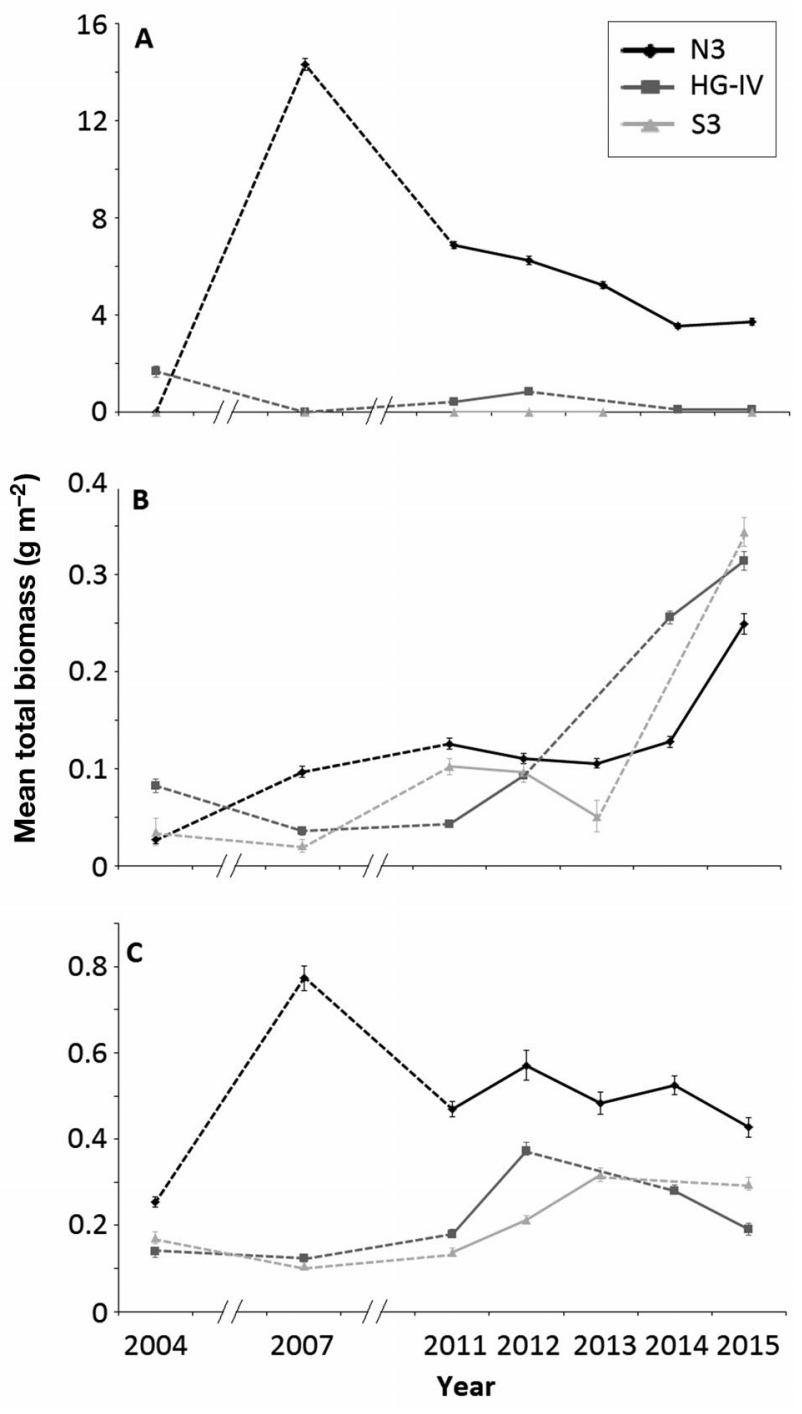

Fig. 5. Mean total biomass of (A) Kolga hyalina, (B) Elpidia heckeri and (C) Mohnia spp. per area $\left(\mathrm{g} \mathrm{m}^{-2}\right)$ calculated for HAUSGARTEN stations N3, HG-IV and S3 for each year. Dashed lines link years between which sampling was not possible. Error bars indicate the standard error of the mean

ations in phospholipids (ANOVA, p $<0.0005, F=$ 8.92, $\mathrm{df}=13$ ) were also observed, with a 6-fold increase between 2014 and 2015 and much higher values than those seen at the other 2 stations (Fig. 6).

\section{DISCUSSION}

\section{Measurement of physical specimens and conversion factors}

Our study is the first estimation of megafaunal biomass at the LTER observatory HAUSGARTEN and, to the authors' knowledge, the first to derive conver-

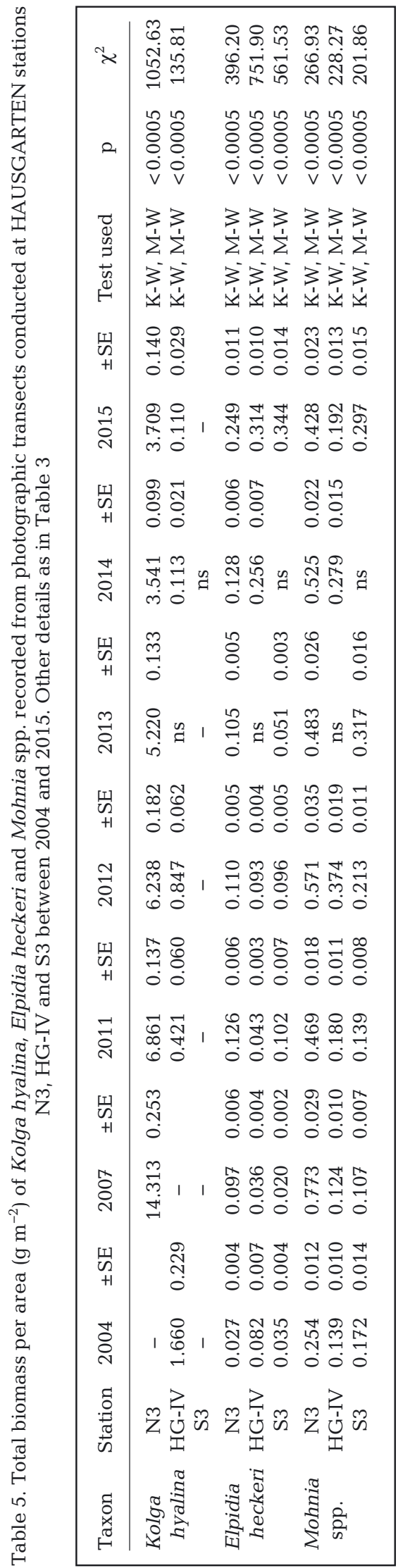



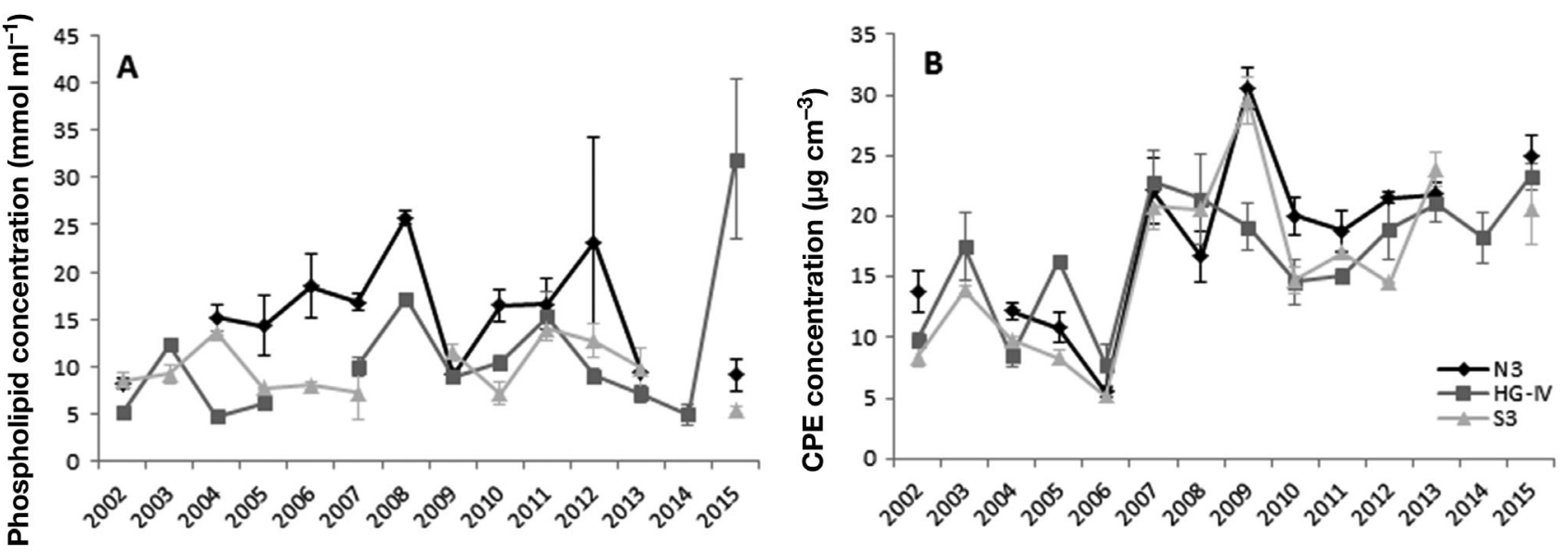

Fig. 6. Mean (A) phospholipid concentrations and (B) chloroplastic pigment equivalents (CPE) from sediments sampled at HAUSGARTEN stations N3 (black diamonds), HG-IV (grey squares) and S3 (light grey triangles) from 2002-2015. Line breaks indicate a lack of data available. Error bars indicate the standard error of the mean

sion factors for the 3 organisms focused on in our investigations. The inclusion of measurements taken of intact physical specimens allowed for conversion of pure length measurements into a measure of biomass, although the conversion factors were derived from relatively small sample sizes, particularly in the case of Elpidia heckeri $(\mathrm{n}=36)$. Still, high adjusted $\mathrm{R}^{2}$ values were obtained for E. heckeri (0.83) and Mohnia spp. (adjusted $\mathrm{R}^{2}=0.86, \mathrm{n}=91$ ), suggesting a good fit of the conversion model. For Kolga hyalina $(\mathrm{n}=186)$, the fit was not as good (adjusted $\mathrm{R}^{2}=0.56$ ). Whilst still adequate, the reduced goodness of fit could be due to factors such as health of individuals/ environment between the 2 trawled years, or differences in the maturity of individuals. Although this is unlikely to play a role in the present study, given the high adjusted $\mathrm{R}^{2}$ values for E. heckeri, the time passed between measurement and capture can have an effect, with up to $40 \%$ total fresh wet weight lost for soft-bodied holothurians (Billett et al. 2001), and therefore cannot be discounted completely as a contributing factor to the derived $\mathrm{R}^{2}$ values despite prompt measurements being taken after specimen collection.

It should also be noted that the method used for measuring individuals in this study (from tip-to-tip) is also a source of potential error, particularly with Kolga hyalina. During feeding, Kolga hyalina may bend their mouth tubes, potentially leading to a $10-20 \%$ underestimation in body length when measured in situ (Rogacheva et. al. 2013). A way to combat this error would be to measure $K$. hyalina from the gonopore to the anus; however, this was not possible in this study as these were not visible in the images at $1.5 \mathrm{~m}$ altitude. Finally, the formally established mod- ern form for describing weight/length relationships (Keys 1928), used in this study, was originally derived for fish. Whilst this study, as well as the studies by Durden et al. $(2015,2016)$, shows that it is a useful tool for estimating biomass of epibenthic, non-fish species, it may not be applicable to all, particularly those with complex body structures.

\section{Temporal biomass estimations}

Our results show that there were temporal variations in the mean mass per individual and the resulting overall biomass of Kolga hyalina, Elpidia heckeri and Mohnia spp. across all stations and years. In addition, our data corroborate the temporal differences in abundances of the 3 studied taxa as shown in Taylor et al. (2017). This indicates that abundance can be considered a valid proxy for overall species biomass of common, non-cryptic species. We found that, for all 3 taxa, as mean mass of an individual increased, the density generally decreased and vice versa. Similar trends were observed at Station $M$, off the central Californian coast, (Ruhl 2007, Huffard et al. 2016). Because of our lack of knowledge of important functional traits such as growth rates, life spans, reproductive gestation periods and migration potential, pinpointing the exact cause is difficult. However, by combining the abundance/mean mass per individual trend with the size frequency distributions we are able to infer whether these changes were weighted towards migration or recruitment. Apart from a few examples, discussed later, the 3 species followed 'normal' reproduction/recruitment cycles. This is clearest in Mohnia spp. as it displayed no 
deviation from the trend. This could be due to their $k$-selected reproductive strategy. Mohnia mohni produces a single embryo, which is supported by thousands of nurse eggs (Bouchet \& Warén 1979), implying high investment in few offspring. This species may therefore not be able to react quickly to favourable environmental conditions, therefore reducing opportunistic breeding potential.

However, given their ability to be able to react quickly to favourable conditions (Huffard et al. 2016), this was not always the case with the holothurians investigated in this study. There were 3 major exceptions to the abundance/mean mass per individual trend being observed: Kolga hyalina at N3 between 2004 and 2007, K. hyalina at HG-IV between 2004 and 2011, and Elpidia heckeri at HG-IV between 2011 and 2015. Two out of these 3 'events' are likely explained best through migration whilst the other indicates a large reproduction/recruitment event followed by rapid growth rates. The first exception, involving E. heckeri in 2012, showed a large increase in the proportion of the smaller size classes, alongside a reduction in mean mass per individual and increase in abundance. From 2012 to 2015, abundance and mean mass per individual increased. This indicates that, although over the years the rate of increase in abundance and mean mass per individual slowed down, the E. heckeri population has not yet reached its carrying capacity, which has increased from 2011 onwards.

The second exception was Kolga hyalina at HG-IV between 2004 and 2011. Whilst occurring at relatively low abundances, the 2 distinct distribution curves in 2004 and 2011, separated by a complete absence of individuals in 2007, suggests either removal via mortality or migration from and then return/ recolonisation at HG-IV. The final exception is $K$. hyalina at N3, between 2004 and 2007. In 2004 there were no $K$. hyalina; however, in the next measured year 3 yr later they were observed in their highest densities, as well as having their highest mean mass per individual. Combining this with there being very few small individuals would lead us to believe this was weighted more towards mass migration and less towards recruitment during this event. Such large 'events' in largely increased numbers have been reported previously for Elpidia glacialis in Antarctica (Gutt et al. 2011), for 4 different holothuroid species (including one Elpidia) (Kuhnz et al. 2014) and for Amperima rosea (Billett et al. 2001, 2010).

Large increases in population density of Amperima rosea was explained as a response to enhanced, localised food inputs, instigating periods of rapid and successful breeding, leading to a large decrease in mean body length (the proxy we used to measure biomass) (Billett et al. 2010). As previously stated, this is not the case with our Kolga hyalina event observations. With our observations during event years, there was a distinct lack of smaller size classes, indicating the absence of juveniles. Whilst it is feasible, given the time between measurements, that $K$. hyalina could have established a population from a nearby area and had a successful reproductive and growth period due to increased food input, continued reproduction would lead to the expected presence of these smaller size classes, particularly in light of the early gonad development and sexual maturity of the closely related $K$. nana (previously described as K. hyalina) (Billett 1988) and slow growth rates in holothurians (Wigham et al. 2003). This further supports the hypothesis of a mass migration event. Previous studies by Billett \& Hansen (1982) and Billett (1991) have shown the opportunistic K. nana to form large aggregations at the Porcupine Seabight off the coast of Ireland, resulting in a patchy distribution, but not to be completely absent then present, or vice versa. Although the $K$. hyalina events at HG-IV and N3 occurred during the same time span, we do not suggest that individuals are migrating the $60 \mathrm{~km}$ between each station. Kaufmann \& Smith (1997) propose $0.4-5.7 \mathrm{~km} \mathrm{yr}^{-1}$ 'walking' speeds for similar sized deep-sea holothurians, meaning this is not plausible in our time frame. Even with the knowledge of the planktonic swimming capability of a closely related species, Kolga nana (Rogacheva et al. 2012), allowing for greater speeds to be feasible, it is still unlikely. In addition, neither of the 2 holothurian species were observed swimming during previous seabed work with remotely operated vehicles, when the vehicle was stationary for extended periods of time at HAUSGARTEN. Instead, as the entire Arctic basin is known to be in the habitable range for $K$. hyalina, we favour individuals migrating/being recruited from nearby populations. To the authors' knowledge, this is the first study to imply a mass migration event in $K$. hyalina.

Both Kolga hyalina events were recorded in 2007 and concur with a 3 to 5 -fold increase in phytodetrital matter at the seafloor (indicated by increased concentrations of sediment-bound chloroplastic pigments) at all stations covered by this study. Between 2005 and 2008, a warm-water anomaly was recorded in the Fram Strait (Beszczynska-M ller et al. 2012), coinciding with reduced sea ice coverage at N3 (Taylor et al. 2017). Also observed in 2007 at N3 was the largest total biomass for Mohnia spp., whereas there was no 
change at HG-IV. Another potential influencing factor in the K. hyalina trends observed was a complete shift in the composition of unicellular plankton organisms at the sea surface with the prymnesiophyte Phaeocystis pouchetii going from absence (pre-2007) to contributing over $90 \%$ of the overall abundance in 2007 (Soltwedel et al. 2016). In our next studied year at HG-IV (2011), the year in which $K$. hyalina returned, this composition had begun to shift away from Phaeocystis dominance. Such large changes in phytoplankton communities could have implications for aggregate size, sedimentation speeds and hence availability of both the quantity and quality of phytodetrital matter at the seafloor. Unfortunately, such data is not available for N3.

\section{SUMMARY AND CONCLUSIONS}

Over the course of the study, we observed temporal differences in total biomass of Kolga hyalina, Elpidia heckeri and Mohnia spp. at all HAUSGARTEN stations investigated, reflecting those observed in abundances, even with varying mean mass per individuals. This result indicates that, at least for these 3 organisms, abundance data may be used as a proxy when discussing total biomass of an organism at a station. This was the first biomass estimations of these 3 organisms to be conducted at HAUSGARTEN, made possible by deriving length to weight conversion factors for these 3 organisms, from physical specimens obtained in 2004, 2005 and 2008.

We were able to distinguish certain life history strategies that each organism employs with the main pattern showing that as abundance goes up the mean mass of an individual goes down, which would seem to indicate regular reproductive/recruitment cycles, a pattern that Mohnia spp. consistently seemed to adopt. Our data showed 3 'events' deviating from this trend, 2 lower-scale events and one large-scale event. The first lower-scale event saw Elpidia heckeri at HG-IV reacting to an undefined factor from 2012 onwards, with rapid reproductive success, likely due to an increased carrying capacity as a result of increased food supply. The second lower-scale event involved the migration/mortality at HG-IV and the return of Kolga hyalina between 2004 and 2011, potentially due to changing particle flux. The largescale event also involved the perceived migration/ recruitment of $K$. hyalina, this time at N3. Migratory numbers were much higher and coincided with a 4 -fold increase in food availability at the seafloor. This is the first time such a large event has been doc- umented for K. hyalina, potentially for all epibenthic deep-sea holothurians.

Our data highlight that as much as community structure and abundance of organisms are constantly changing in deep-sea epibenthic communities (Taylor et al. 2017), so is biomass. To maximise our understanding of the life history strategies of these relatively inaccessible organisms, we should use all tools available. Only through the combination of abundance, mean mass per individual and relative size distributions were we able to explore potential mechanisms at play, which currently evade us for much of the deep sea. Climate models provided by Henson et al. (2016) suggest that $\sim 30 \mathrm{yr}$ of observations are needed to be able to distinguish climate change trends from natural variability in the Arctic. The nature of sustained long-term ecological research at HAUSGARTEN allows for this to be achieved, further unravelling key factors and influences on deepsea epibenthic communities.

Acknowledgements. We thank the officers, crew and principle scientists of RV 'Polarstern' for their assistance at sea during the expeditions: ARK-XX/1, ARK-XXII/1, ARK-VI/2, ARK-XXVII/2, ARK-XXVIII/1 and ARK-XXIX/2, as well as those of RV 'Maria S. Merian' during the expedition MSM29. We also thank all scientists and technical staff who operated and maintained the OFOS. C. Hasemann and I. Schewe were in charge of multiple corer deployment and sediment analyses assisted by A. Pappert and numerous volunteers and students. We thank A. Warén (Swedish Museum of Natural History) for assistance with Mohnia spp. physical specimen identification and G. Schmidt for assistance with image analysis. D. Langenkämper (Universität Bielefeld) provided user support with BIIGLE. J.T. was funded by a DAAD PhD scholarship and the EU-FP7 project FixO3 - Fixed-point Open Ocean Observatories (grant agreement no. 312463). M.B. was funded by the Helmholtz Alliance ROBEX. The study contributes to the tasks 4.2 .2 of the Helmholtz-funded infrastructure programme FRAM (Frontiers in Arctic Marine Research). This publication is Eprint ID 45790 of the Alfred-Wegener-Institut, Helmholtz-Zentrum für Polar- und Meeresforschung.

\section{LITERATURE CITED}

Alt CHS, Rogacheva A, Boormann B, Hughes JA, Billett DSM, Gooday AJ, Jones DOB (2013) Trawled megafaunal invertebrate assemblages from bathyal depth of the Mid-Atlantic Ridge $\left(48^{\circ}-54^{\circ} \mathrm{N}\right)$. Deep-Sea Res II 98: 326-340

Bauerfeind E, Nöthig EM, Beszczynska A, Fahl K and others (2009) Particle sedimentation patters in the eastern Fram Strait during 2000-2005: results from the Arctic longterm observatory HAUSGARTEN. Deep-Sea Res I 56: 1471-1487

*Bergmann M, Dannheim J, Bauerfeind E, Klages M (2009) Trophic relationships along a bathymetric gradient at the 
deep-sea observatory HAUSGARTEN. Deep-Sea Res I 56:408-424

Bergmann M, Soltwedel T, Klages M (2011) The interannual variability of megafaunal assemblages in the Arctic deep sea: preliminary results from the HAUSGARTEN observatory $\left(79^{\circ} \mathrm{N}\right)$. Deep-Sea Res I 58:711-723

Bernard BB, Bernard H, Brooks JM (2004) Determination of total carbon, total organic carbon and inorganic carbon in sediments. TDI-Brooks International/B\&B Laboratories, College Station, TX

Beszczynska-M ller A, Fahrbach E, Schauer U, Hansen E (2012) Variability in Atlantic water temperature and transport at the entrance to the Arctic Ocean, 1997-2010. ICES J Mar Sci 69:852-863

Billett DSM (1988) The ecology of deep-sea holothurians. $\mathrm{PhD}$ dissertation, University of Southampton

Billett DSM (1991) Deep-sea holothurians. Oceanogr Mar Biol Annu Rev 29:259-317

Billett DSM, Hansen B (1982) Abyssal aggregations of Kolga hyalina Danielssen and Korén (Echinodermata: Holothurioidea) in the northeast Atlantic Ocean: a preliminary report. Deep-Sea Res A 29:799-818

* Billett DSM, Bett BJ, Rice AL, Thurston MH, Galéron J, Sibuet M, Wolff GA (2001) Long-term change in the megabenthos of the Porcupine Abyssal Plain (NE Atlantic). Prog Oceanogr 50:325-348

* Billett DSM, Bett BJ, Reid WDK, Boorman B, Priede IG (2010) Long-term change in the abyssal NE Atlantic: the 'Amperima Event' revisited. Deep-Sea Res II 57: 1406-1417

Bouchet P, Warén A (1979) The abyssal molluscan fauna of the Norwegian Sea and its relation to other faunas. Sarsia 64:211-243

Buhl-Mortensen L, Tandberg AHS, Buhl-Mortensen P, Gates AR (2016) Behaviour and habitat of Neohela monstrosa (Boeck, 1861) (Amphipoda: Corophiida) in Norwegian Sea deep water. J Nat Hist 50:323-337

* Durden JM, Bett BJ, Jones DOB, Huvenne VAI, Ruhl HA (2015) Abyssal hills hidden sources of increased habitat heterogeneity, benthic megafaunal biomass and diversity in the deep sea. Prog Oceanogr A 137: 209-218

* Durden JM, Bett BJ, Horton T, Serpell-Stevens A, Morris KJ, Billett DSM, Ruhl HA (2016) Improving the estimation of deep-sea megabenthos biomass: dimension to wet weight conversions for abyssal invertebrates. Mar Ecol Prog Ser 552:71-79

Findlay RH, King GM, Watling L (1989) Efficiency of phospholipid analysis in determining microbial biomass in sediments. Appl Environ Microbiol 55:2888-2893

FitzGeorge-Balfour T, Billett DSM, Wolff GA, Thompson A, Tyler PA (2010) Phytopigments as biomarkers of selectivity in abyssal holothurians; interspecific differences in response to a changing food supply. Deep-Sea Res II 57: $1418-1428$

Forest A, Wassmann P, Slagstad D, Bauerfeind E, Nöthig EM, Klages M (2010) Relationships between primary production and vertical particle export at the AtlanticArctic boundary (Fram Strait, HAUSGARTEN). Polar Biol 33:1733-1746

* Górska B, Grzelak K, Kotwicki L, Hasemann C, Schewe I, Soltwedel T, Wlodarska-Kowalczuk M (2014) Bathymetric variations in vertical distribution patterns of meiofauna in the surface sediments of the deep Arctic ocean (HAUSGARTEN, Fram Strait). Deep-Sea Res I 91:36-49 * Grassle JF, Sanders HL, Hessler RR, Rowe GT, McLellan T
(1975) Pattern and zonation: a study of the bathyal megafauna using the research submersible Alvin. DeepSea Res Oceanogr Abstr 22:457-462

Greiser N, Faubel A (1988) Biotic factors. In: Higgens RP, Thiel $\mathrm{H}$ (eds) Introduction to the study of meiofauna. Smithsonian Institution Press, Washington, DC, p 79-114

*Gutt J, Barratt I, Domack E, d'Udekem d'Acoz C and others (2011) Biodiversity change after climate-induced iceshelf collapse in the Antarctic. Deep-Sea Res II 58:74-83

*Hargrave BT, Kostylev VE, Hawkins CM (2004) Benthic epifauna assemblages, biomass and respiration in the Gully region on the Scotian Shelf, NW Atlantic Ocean. Mar Ecol Prog Ser 270:55-70

*Hasemann C, Soltwedel T (2011) Small-scale heterogeneity in deep-sea nematode communities around biogenic structures. PLOS ONE 6:e29152

*Henson SA, Beaulieu C, Lampitt R (2016) Observing climate change trends in ocean biogeochemistry: when and where. Glob Change Biol 22:1561-1571

* Huffard CL, Kuhnz LA, Lemon L, Sherman AD, Smith KL Jr (2016) Demographic indicators of change in a depositfeeding abyssal holothurian community (Station M, 4000 m). Deep-Sea Res I 109:27-39

Jacob M, Soltwedel T, Boetius A, Ramette A (2013) Biogeography of deep-sea benthic bacteria at regional scale (LTER HAUSGARTEN, Fram Strait, Arctic). PLOS ONE 8:e72779

Kaufmann RS, Smith KL (1997) Activity patterns of mobile epibenthic megafauna at an abyssal site in the eastern North Pacific: results from a 17-month time-lapse photographic study. Deep-Sea Res I 44:559-579

Keys AB (1928) The weight-length relation in fishes. Proc Natl Acad Sci USA 14:922-925

Kuhnz LA, Ruhl HA, Huffard CL, Smith KL Jr (2014) Rapid changes and long-term cycles in the benthic megafaunal community observed over 24 years in the abyssal northeast Pacific. Prog Oceanogr 124:1-11

Lalande C, Bauerfeind E, Nöthig EM, Beszczynska-Möller A (2013) Impact of a warm anomaly on export fluxes of biogenic matter in the eastern Fram Strait. Prog Oceanogr 109:70-77

* Langenkämper D, Zurowietz M, Schoening T, Nattkemper TW (2017) BIIGLE 2.0 - Browsing and annotating large marine image collections. Front Mar Sci 4:83

* Meyer KS, Bergmann M, Soltwedel T (2013) Interannual variation in the epibenthic megafauna at the shallowest station of the HAUSGARTEN observatory $\left(79^{\circ} \mathrm{N}, 6^{\circ} \mathrm{E}\right)$. Biogeosciences 10:3479-3492

* Meyer KS, Young CM, Sweetman AK, Taylor J, Soltwedel T, Bergmann M (2016) Rocky islands in a sea of mud: biotic and abiotic factors structuring deep-sea dropstone communities. Mar Ecol Prog Ser 556:45-57

* Piepenburg D, Schmid MK (1996) Distribution, abundance, biomass, and mineralization potential of the epibenthic megafauna of the Northeast Greenland shelf. Mar Biol 125:321-332

* Ramírez-Llodra E, Ballesteros M, Company JB, Dantart L, Sardà F (2007) Spatio-temporal variations of biomass and abundance in bathyal non-crustacean megafauna in the Catalan Sea (north-western Mediterranean). Mar Biol 153:297-309

Rogacheva A, Gebruk A, Alt CHS (2012) Swimming deepsea holothurians (Echinodermata: Holothuroidea) on the northern Mid-Atlantic Ridge. Zoosymposia 224:213-224

Kogacheva A, Gebruk A, Alt CHS (2013) Holothuroidea of 
the Charlie Gibbs Fracture Zone area, northern MidAtlantic Ridge. Mar Biol Res 9:587-623

Rowe GT, Wei C, Nunnally C, Haedrich R and others (2008) Comparative biomass structure and estimated carbon flow in food webs in the deep Gulf of Mexico. Deep-Sea Res II 55:2699-2711

Ruhl HA (2007) Abundance and size distribution dynamics of abyssal epibenthic megafauna in the northeast Pacific. Ecology 88:1250-1262

Ruhl HA, Bett BJ, Hughes SJM, Alt CHS and others (2014) Links between deep-sea respiration and community dynamics. Ecology 95:1651-1662

Soltwedel T, Bauerfeind E, Bergmann M, Bracher A and others (2016) Natural variability of anthropogenicallyinduced variation? Insights from 15 years of multidisciplinary observations at the Arctic marine LTER site HAUSGARTEN. Ecol Indic 65:89-102

Taylor J, Krumpen T, Soltwedel T, Gutt J, Bergmann M (2016) Regional- and local-scale variations in benthic megafaunal composition at the Arctic deep-sea observatory HAUSGARTEN. Deep-Sea Res I 108:58-72

Editorial responsibility: James McClintock, Birmingham, Alabama, USA
Taylor J, Krumpen T, Soltwedel T, Gutt J, Bergmann M (2017) Dynamic benthic megafaunal communities: assessing temporal variations in structure, composition and diversity at the Arctic deep-sea observatory HAUSGARTEN between 2004 and 2015. Deep-Sea Res I 122:81-94

* Van Oevelen D, Soetaert K, Heip C (2012) Carbon flows in the benthic food web of the Porcupine Abyssal Plain: The (un)importance of labile detritus in supporting microbial and faunal carbon demands. Limnol Oceanogr 57: 645-664

Von Appen WJ, Schauer U, Somavilla-Cabrillo R, Bauerfeind E, Beszczynska-Möller A (2015) Exchange of warming deep waters across Fram Strait. Deep-Sea Res I 103:86-100

*Wigham BD, Tyler PA, Billett DSM (2003) Reproductive biology of the abyssal holothurian Amperima rosea: an opportunistic response to variable flux of surface derived organic matter? J Mar Biol Assoc UK 83:175-188

Y Yentsch CS, Menzel DW (1963) A method for the determination of phytoplankton chlorophyll and phaeophytin by fluorescence. Deep-Sea Res Oceanogr Abstr 10:221-231

Submitted: November 7, 2017; Accepted: June 1, 2018 Proofs received from author(s): August 6, 2018 